\title{
Improved Single-Key Attacks on 8-Round AES-192 and AES-256
}

\author{
Orr Dunkelman, Nathan Keller*, and Adi Shamir \\ Faculty of Mathematics and Computer Science \\ Weizmann Institute of Science \\ P.O. Box 26, Rehovot 76100, Israel \\ \{orr.dunkelman, nathan.keller, adi.shamir\}@weizmann.ac.il
}

\begin{abstract}
AES is the most widely used block cipher today, and its security is one of the most important issues in cryptanalysis. After 13 years of analysis, related-key attacks were recently found against two of its flavors (AES-192 and AES-256). However, such a strong type of attack is not universally accepted as a valid attack model, and in the more standard single-key attack model at most 8 rounds of these two versions can be currently attacked. In the case of 8-round AES-192, the only known attack (found 10 years ago) is extremely marginal, requiring the evaluation of essentially all the $2^{128}$ possible plaintext/ciphertext pairs in order to speed up exhaustive key search by a factor of 16 . In this paper we introduce three new cryptanalytic techniques, and use them to get the first non-marginal attack on 8-round AES-192 (making its time complexity about a million times faster than exhaustive search, and reducing its data complexity to about $1 / 32,000$ of the full codebook). In addition, our new techniques can reduce the best known time complexities for all the other combinations of 7-round and 8-round AES-192 and AES-256.
\end{abstract}

\section{Introduction}

The Rijndael block cipher [6] was developed in the late 1990's by Joan Daemen and Vincent Rijmen, and was selected as the Advanced Encryption Standard (AES) in 2001. Over the last ten years it replaced the Data Encryption Standard (DES) in most applications, and had become the block cipher of choice for any new security application. It has three possible key sizes (128, 192, and 256 bits), and in 2003 the US government had publicly announced that AES-128 can be used to protect classified data up to the level of "secret", and that AES-192 and AES-256 can be used to protect classified data up to the level of "top secret".

Due to its importance and popularity, the security of AES had attracted a lot of attention, and is considered one of the hottest areas of research in cryptanalysis. A major breakthrough was the recent discovery of related-key attacks on the full versions of AES-192 and AES-256 34 which are faster than exhaustive search, but have impractical complexities. In another line of research [2],

\footnotetext{
* The second author was partially supported by the Koshland center for basic research.
} 
related-key attacks requiring practical time complexity of $2^{45}$ were found on AES-256 with up to 10 rounds, and related key attacks requiring semipractical time complexity of $2^{70}$ were found on AES-256 with 11 rounds.

The main weakness of AES-192 and AES-256 exploited in these attacks was their extremely simple key schedule. In a related-key attack model, this made it possible to cancel data differences with corresponding key differences over many rounds of AES. This created a very high probability differential characteristic, which led to a greatly improved time complexity. However, such attacks make a very strong assumption that the adversary can ask the encryption box to modify the unknown key in a known way. Some of these attacks even assume that the adversary can obtain a large number of related keys, or that he can obtain related intermediate subkeys — see [3] for a discussion of these possibilities. Consequently, related-key attacks are important considerations during the design and certification stage of new ciphers, but are not considered a realistic threat in practical security protocols which use the block cipher in a standard way.

In this paper we consider the classical attack model of a single key and multiple known or chosen plaintext/ciphertext pairs. In this model the adversary has to deal with the very well designed data path of AES, and cannot directly benefit from its weak key schedule. Consequently, there are no known attacks on the full cipher on any one of the three flavors of AES, and the best we can do is to attack reduced round versions of AES. In the case of AES-256, the largest number of rounds that can be attacked is 8 . In the case of AES-192 there is one attack on 8-round AES-192 which was published in [10, it is extremely marginal: It requires the evaluation of essentially all the possible plaintext/ciphertext pairs under the unknown key, and even then the time required to derive the key is only 16 times faster than exhaustive search (one can argue that given the complete codebook of size $2^{128}$, there is no need to find the actual key in order to easily decrypt any given ciphertext ...). In the case of AES-128, there are no known attacks on its 8-round version, but there are a few on 7 -round variants.

In order to improve all these known attacks, and especially the marginal attack on 8-round AES-192 which no one was able to improve upon in the last ten years, we develop three new cryptanalytic techniques. Our starting point is the attack on 7-round AES developed by Gilbert and Minier [11, which constructs a large table of $2^{72}$ entries, where each entry contains a sequence of 256 byte values. This idea was extended to 8-round AES by Demirci and Selçuk [7], who constructed an even larger table of $2^{192}$ entries (again containing sequences of 256 byte values, which are constructed in a slightly modified way). Due to the $2^{200}$ time required just to construct this table, this attack is worse than exhaustive search for 8-round AES-192, and can only be applied to 8-round AES-256.

Our first new idea (called multiset tabulation) is to replace the sequence of 256byte values in each table entry by the multiset of its values. Even though we lose some information, we show that it is still possible to use such a table in order to discard with very high probability incorrect key guesses. This modification makes it possible to reduce the number of table entries (and thus also the time required to prepare the table) by a factor of $2^{16}$. An even bigger saving (by a factor of 
$2^{57}$ ) in the size of the table is obtained by another new technique which we call differential enumeration. It uses some truncated differential (which need not have particularly high or low probability, as required in standard or impossible differential attacks) in order to enumerate the entries of such a table in a much more efficient way: Instead of directly enumerating state values, the adversary derives them indirectly by enumerating the input and output differential values of certain internal S-boxes. By reducing the space complexity in such a major way, we can now trade it off with the high time complexity of the Demirci and Selçuk attack in order to greatly improve it. Finally, we develop a new key bridging technique which exploits the weak key schedule of AES by using the following surprising observation: In the particular case of 8-round AES-192, it is possible to compute one byte of the whitening subkey (used before the first round) directly from four bytes of the last subkey (used at the end of the eighth round), despite their distance. Since our attack requires guessing these five subkey bytes in the first and last rounds, we get an extra savings of $2^{8}$ in our time complexity 1 By combining these three techniques, we can now break 8-round AES-192 in about one millionth of the complexity of exhaustive search.

Our new results are summarized and compared with previously known singlekey attacks in Table 1. As can be seen, our time complexities for 8-round AES are considerably better than the best previous results for both AES-192 and AES-256.

This paper is organized as follows: In Section 2 we describe the AES block cipher and introduce our notations. In Section 3 we describe the techniques used in previous attacks, and analyze their complexity. In Section 4 we introduce our new cryptanalytic techniques. We use them in Section 5 to improve the best known attacks on 7-round AES, and in Section [6 to improve the best known attacks on 8-round AES. Finally, in Section 7 we summarize our results.

\section{A Short Description of AES}

The advanced encryption standard (AES) [6] is an SP-network that supports key sizes of 128,192 , and 256 bits. A 128-bit plaintext is treated as a byte matrix of size $4 x 4$, where each byte represents a value in $G F\left(2^{8}\right)$. An AES round applies four operations to the state matrix:

- SubBytes (SB) - applying the same 8-bit to 8-bit invertible S-box 16 times in parallel on each byte of the state,

- ShiftRows (SR) - cyclically shifting the $i$ 'th row by $i$ bytes to the left,

- MixColumns (MC) - multiplication of each column by a constant $4 \times 4$ matrix over the field $G F\left(2^{8}\right)$, and

- AddRoundKey (ARK) - XORing the state with a 128-bit subkey.

We outline an AES round in Figure 1.

${ }^{1}$ The same idea can be used to improve the time complexity of several other attacks such as 10[14 by the same factor of $2^{8}$. 


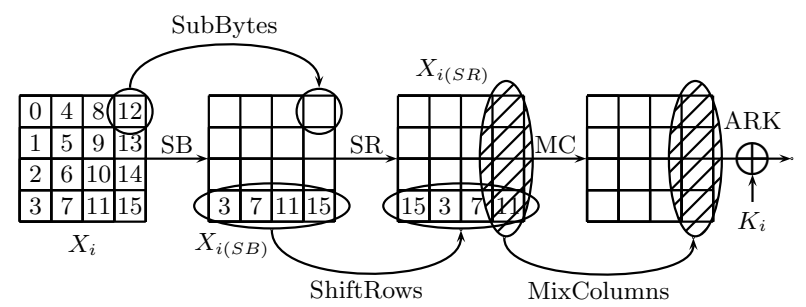

Fig. 1. An AES round

In the first round, an additional AddRoundKey operation (using a whitening subkey) is applied, and in the last round the MixColumns operation is omitted. Rounds which include the MixColumns operation are called full rounds.

The number of rounds depends on the key length: 10 rounds for 128-bit keys, 12 rounds for 192-bit keys, and 14 rounds for 256-bit keys. The rounds are numbered $0, \ldots, N r-1$, where $N r$ is the number of rounds. For the sake of simplicity we shall denote AES with $n$-bit keys by AES- $n$, e.g., AES with 128 bit keys (and thus with 10 rounds) is denoted by AES-128. We use AES to mean all three variants of AES.

The key schedule of AES takes the user key and transforms it into $\mathrm{Nr}+1$ subkeys of 128 bits each. The subkey array is denoted by $W[0, \ldots, 4 \cdot N r+3]$, where each word of $W[\cdot]$ consists of 32 bits. Let the length of the key be $N k$ 32-bit words, then the first $N k$ words of $W[\cdot]$ are loaded with the user supplied key. The remaining words of $W[\cdot]$ are updated according to the following rule:

- For $i=N k, \ldots, 4 \cdot N r+3$, do

- If $i \equiv 0 \bmod N k$ then $W[i]=W[i-N k] \oplus S B(W[i-1] \lll 8) \oplus$ $R C O N[i / N k]$,

- else if $N k=8$ and $i \equiv 4 \bmod 8$ then $W[i]=W[i-8] \oplus S B(W[i-1])$,

- Otherwise $W[i]=W[i-1] \oplus W[i-N k]$,

where $R C O N[\cdot]$ is an array of predetermined constants, and $\lll$ denotes rotation of the word by 8 bits to the left.

\subsection{The Notations Used in the Paper}

In the sequel we use the following definitions and notations: The state matrix at the beginning of round $i$ is denoted by $X_{i}$, and its bytes are denoted by $0,1,2, \ldots, 15$, as described in Figure 1. Similarly, the state matrix after the SubBytes and the ShiftRows operations of round $i$ are denoted by $X_{i(S B)}$ and $X_{i(S R)}$, respectively.

We denote the subkey of round $i$ by $k_{i}$, and the first (whitening) key by $k_{-1}$, i.e., $k_{i}=W[4 \cdot(i+1)]\|W[4 \cdot(i+1)+1]\| W[4 \cdot(i+1)+2] \| W[4 \cdot(i+1)+3]$. In some cases, we are interested in interchanging the order of the MixColumns operation and the subkey addition. As these operations are linear they can be interchanged, by first XORing the data with an equivalent subkey and only 
then applying the MixColumns operation. We denote the equivalent subkey for the altered version by $u_{i}$, i.e., $u_{i}=M C^{-1}\left(k_{i}\right)$. The bytes of the subkeys are numbered by $0,1, \ldots, 15$, in accordance with the corresponding state bytes.

We use the following notations for intermediate encryption values: The intermediate state at the beginning of round $i$ in the encryption of $P^{j}$ is denoted by $X_{i}^{j}$, and its bytes are denoted by $X_{i, l}^{j}$, for $0 \leq l \leq 15$. Similarly, the intermediate values after the SubBytes and the ShiftRows operations of round $i$ are denoted by $X_{i(S B), l}^{j}$ and $X_{i(S R), l}^{j}$, respectively.

In our attacks we mostly consider the encryption of $\delta$-sets, which are structured sets of 256 plaintexts $\left\{P^{0}, P^{1}, \ldots, P^{255}\right\}$ in which one active byte assumes each one of the 256 possible values exactly once, and each one of the other 15 bytes is a (possibly different) constant. A state byte is called balanced if the XOR of its 256 values during the encryption of a $\delta$-set is zero.

In all the observations considering reduced-round versions of AES, the numbering of the rounds starts with round 0 . When we analyze the behavior of some consecutive inner rounds of AES, we shift the round numbering accordingly, depending on the number of rounds we add at the beginning.

Finally, we measure the time complexity of all the attacks in units which are equivalent to a single encryption operation of the relevant reduced round variant of AES. We measure the space complexity in units which are equivalent to the storage of a single plaintext (namely, 128 bits). To be completely fair, we count all operations carried out during our attacks, and in particular we do not ignore the time and space required to prepare the various tables we use.

\section{Previous Work}

The first attack developed against AES was the SQUARE attack, which was found by its designers [5]. The SQUARE attack is based on:

Observation 1. Consider the encryption of a $\delta$-set through three full AES rounds. The set of 256 corresponding ciphertexts is balanced, i.e., the XOR of the 256 values in each one of its 16 bytes is zero.

The observation follows easily from the structure of AES, as demonstrated in Figure 2. This property is the basis of many attacks on reduced round variants of AES. The original submission [5] offers a 6-round attack with time complexity of $2^{72}$, which was later improved in [10] using the partial sums technique to offer the best known attack on 6-round AES (with time $2^{42}$ ).

In [1], Gilbert and Minier proposed to refine the information on the intermediate encryption values of the $\delta$-sets exploited in the SQUARE attack. Their attack is based on the following observation:

Observation 2. Consider the encryption of a $\delta$-set through three full AES rounds. For each one of the 16 bytes of the ciphertext, we can define a sequence of 256 values for this byte by ordering the plaintexts according to the value of their active byte. Then any such sequence is fully determined by just 9 bytes, which are complex functions of the constants in the $\delta$-set and the key bytes. Consequently, for 


\begin{tabular}{|c|c|c|c|c|c|c|c|c|c|c|c|c|c|c|c|c|c|c|c|c|c|c|}
\hline \begin{tabular}{|l|}
$\mathcal{A}$ \\
\end{tabular} & $\mathcal{C}$ & $\mathcal{C}$ & & & \begin{tabular}{|l}
$\mathcal{A}$ \\
\end{tabular} & $\mathcal{C}$ & $\mathcal{C}$ & & & $\mathcal{A}$ & & \begin{tabular}{|l|}
$\mathcal{C}$ \\
\end{tabular} & & & $\mathcal{C}$ & $\mathcal{C}$ & & & $c$ & $\mathcal{C}$ & & \\
\hline $\mathcal{C}$ & $\overline{\mathcal{C}}$ & $\mathcal{C}$ & $\overline{\mathcal{C}}$ & $\mathrm{SB}$ & $\mathcal{C}$ & $\mathcal{C}$ & $c$ & $\mathcal{C}$ & $\mathrm{MC}$ & $\bar{A}$ & $\mathcal{C}$ & $c$ & 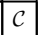 & $\mid \mathcal{A}$ & $\mathcal{C}$ & \begin{tabular}{l|l|l}
$\mathcal{C}$ & \\
\end{tabular} & SB & C & $\mathcal{C}$ & 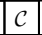 & $\overline{\mathcal{A}}$ & $\mathrm{MC}$ \\
\hline $\mathcal{C}$ & $\overline{\mathcal{C}}$ & $\mathcal{C}$ & $\overline{\mathcal{C}}$ & SR & $\mathcal{C}$ & $\mathcal{C}$ & $\mathcal{C}$ & $\mathcal{C}$ & & $\bar{A}$ & $\mathcal{C}$ & $\mathcal{C}$ & $\mathcal{C}$ & $\mathcal{A}$ & \begin{tabular}{c|}
$\mathcal{C}$ \\
\end{tabular} & \begin{tabular}{l|l}
$\mathcal{C}$ & \\
\end{tabular} & $\begin{array}{l}\text { SR } \\
\end{array}$ & $\mathcal{C}$ & $\mathcal{C}$ & & $\overline{\mathcal{C}}$ & \\
\hline \begin{tabular}{|c|}
$\mathcal{C}$ \\
\end{tabular} & $\mathcal{C}$ & $\mathcal{C}$ & $\mathcal{C}$ & & $\begin{array}{ll}\mathcal{C} \\
\end{array}$ & $\mathcal{C}$ & $\mathcal{C}$ & $\mathcal{C}$ & & $\mathcal{A}$ & $\mathcal{C}$ & $\mathcal{C}$ & $\mathcal{C}$ & & $\mathcal{C}$ & \begin{tabular}{l|l} 
& \\
\end{tabular} & & & $\mathcal{A}$ & $\mathcal{C}$ & $\mathcal{C}$ & \\
\hline
\end{tabular}

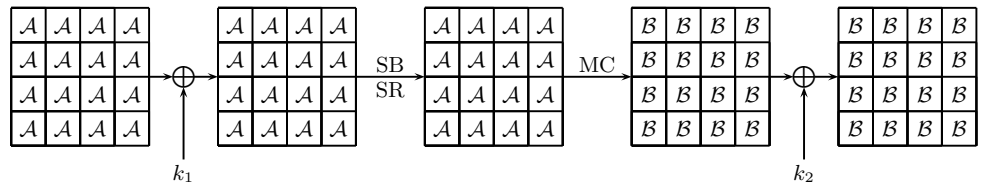

Fig. 2. The development of a $\delta$-set through 3 rounds of AES, where $\mathcal{A}$ stands for an active byte, $\mathcal{B}$ stands for a balanced byte, and $\mathcal{C}$ stands for a constant byte

any fixed byte position, there are at most $2^{72}$ possible sequences when we consider all the possible choices of keys and $\delta$-sets (out of the $\left(2^{8}\right)^{256}=2^{2048}$ of the "theoretically possible" 256-byte sequences, and out of the $2^{256+15 \times 8}=2^{376}$ sequences which could be potentially defined by the choice of 15 constant bytes and 256 key bits.)

This observation was used in 11] to mount an attack on 7-round AES-128 with time complexity slightly smaller than that of exhaustive key search. Since the attack algorithm is a bit complex and not used in our paper, we omit it here.

In [7], Demirci and Selçuk extended the observation of [11] by another round. They showed the following:

Observation 3. Consider the encryption of a $\delta$-set through four full AES rounds. For each of the 16 bytes of the state, the ordered sequence of 256 values of that byte in the corresponding ciphertexts is fully determined by just 25 byte parameters. Consequently, for any fixed byte position, there are at most $2^{200}$ possible sequences when we consider all the possible choices of keys and $\delta$-sets 2

This observation was used in [7] to mount attacks on 7-round and 8-round variants of AES-256. The attack on 7-round AES-256 is roughly as follows:

1. Preprocessing phase: Compute all the $2^{192}$ possible values of the 255 -byte sequence given in Observation 3, and store them in a hash table.

2. Online phase:

(a) Guess the value of four bytes in the whitening key $k_{-1}$ and of one byte in $k_{0}$, and for each guess, construct a $\delta$-set from the data. (For example, if the active byte of the $\delta$-set is byte 0 , then the guessed bytes are bytes

${ }^{2}$ In [7] the authors note that the function $f_{c_{1}, \ldots, c_{25}}(x)$ can be written as $f_{c_{1}, \ldots, c_{25}}(x)=$ $g_{c_{1}, \ldots, c_{24}}(x) \oplus c_{25}$, and thus one can reduce the number of possible sequences by picking some $x_{0}$, and considering the augmented function $f_{c_{1}, \ldots, c_{24}}^{\prime}(x)=f_{c_{1}, \ldots, c_{25}}(x)-$ $f_{c_{1}, \ldots, c_{25}}\left(x_{0}\right)=g_{c_{1}, \ldots, c_{24}}(x)-g_{c_{1}, \ldots, c_{24}}\left(x_{0}\right)$. In this case, the number of parameters is reduced to 24 , the number of "interesting" entries in each sequence is reduced to 255 (as $f^{\prime}\left(x_{0}\right)=0$, independently of the choice of $x_{0}$ and $c_{1}, \ldots, c_{24}$ ), and the number of possible sequences is reduced to $2^{192}$. 
$0,5,10,15$ of $k_{-1}$ and byte 0 of $k_{0}$. Note that byte 0 of $k_{0}$ is used only to compute the order of the values in the $\delta$-set).

(b) Guess four bytes of the equivalent subkey $u_{6}$ and one byte of the equivalent subkey $u_{5}$ and partially decrypt the ciphertexts of the $\delta$-set to obtain the sequence of 256 intermediate values of one byte of the state $X_{5}$. (For example, if the byte to be checked is byte 0 , then the subkey bytes the adversary should guess are byte 0 of $u_{5}$ and bytes $0,7,10,13$ of $u_{6}$ ).

(c) Check whether the sequence exists in the hash table. If not, discard the key guess.

The data complexity of the attack is $2^{32}$ chosen plaintexts. The time complexity of the online phase is relatively modest at $2^{80}$, but the space complexity and the time complexity in encryption operations required to prepare this large table are about $2^{200}$. These complexities are worse than exhaustive search for both AES-192 and AES-128. However, Demirci and Selçuk presented a tradeoff, which makes it possible to decrease the memory complexity at the expense of increasing both the data and the online time complexities. This results in an attack on 7 round AES-192 with data complexity of $2^{96}$ chosen plaintexts, and time and space complexities of $2^{144}$.

The attack in 7] can be extended to 8-round AES-256 by guessing the full subkey of the last round. This increases the time complexity of the online phase from $2^{80}$ to $2^{208}$ encryptions, and makes it impossible to rebalance the parameters in order to attack 8-round AES-1923.

\section{Our New Techniques}

In this section we present three new techniques. First, we present a new variant of Observation 3 which is stronger and simpler to analyze. Then we show how a combination of the $\delta$-set analysis with a 4 -round differential allows to reduce the memory complexity of the attack by a factor of $2^{57}$. Finally, we show that for AES-192 and AES-256, the time complexity of the 8-round attack can be reduced using key schedule considerations by a factor of $2^{32}$ and $2^{8}$, respectively.

\subsection{The Multiset Variant of the Demirci-Selçuk Observation}

We start with our new variant of Observation 3 .

Observation 4. Consider the encryption of a $\delta$-set $\left\{P^{0}, P^{1}, \ldots, P^{255}\right\}$ through four full AES rounds.

\footnotetext{
${ }^{3}$ We note that in a more recent paper, Demirci et al. 8] claim that by optimizing their technique they can also attack 7-round AES-128 faster than exhaustive search. However, we note that the analysis of 8 is flawed, and the correct running time of the attack is about $2^{32}$ times more than claimed, and in particular more than the complexity of exhaustive key search for the 128-bit key version.
} 
For each $0 \leq l \leq 15$, the (un-ordered) multise 4 $\left[X_{4, l}^{0} \oplus X_{4, l}^{0}, X_{4, l}^{1} \oplus X_{4, l}^{0}, \ldots, X_{4, l}^{255} \oplus X_{4, l}^{0}\right]$ is fully determined by the following 24 byte parameters:

- The full 16-byte state $X_{2}^{0}$.

- Four bytes of the state $X_{1}^{0}$. (For example, if the active byte of the $\delta$-set is byte 0 then these are bytes $0,1,2,3)$.

- Four bytes of the subkey $k_{2}$. (For example, if $l=0$ then these are bytes $0,5,10,15)$.

Moreover, this multiset can assume only $2^{184}$ values (out of the $\left(\begin{array}{l}511 \\ 256\end{array}\right) \approx 2^{507.6}$ "theoretically possible" values).

Our variant has several advantages over Observation 3 ;

- The parameters upon which the sequence depends are specified explicitly. This is crucial for the major reduction in the number of parameters presented in the next section.

- The smaller number of possible configurations in our variant $\left(2^{184}\right.$ instead of $2^{192}$ ) allows to reduce the memory requirements of the attack and the time complexity of the preprocessing phase by a factor of $2^{8}$.

- Since we consider a multiset instead of an ordered sequence, the adversary does not need to know the order of the values in the $\delta$-set at the beginning of the four rounds. This allows to skip the guess of one byte in the subkey $k_{0}$ (reducing the time complexity of the online phase by $2^{8}$ ).

Proof . The proof emphasizes the meet-in-the-middle nature of the observation.

We start with the "bottom side" of the four rounds. First, we observe that if $\left\{X_{2}^{0}, X_{2}^{1}, \ldots, X_{2}^{255}\right\}$ are known, then the knowledge of bytes $0,5,10,15$ of $k_{2}$ yields the knowledge of the entire first column before the AddRoundKey of round 3 in all the 256 encryptions. Since the AddRoundKey preserves differences, this yields the desired values of the vector of differences $\left(X_{4, l}^{0} \oplus X_{4, l}^{0}, X_{4, l}^{1} \oplus X_{4, l}^{0}, \ldots, X_{4, l}^{255} \oplus X_{4, l}^{0}\right)$.

Secondly, to know the values $\left\{X_{2}^{0}, X_{2}^{1}, \ldots, X_{2}^{255}\right\}$, it is sufficient to know the value $X_{2}^{0}$ which is given as part of the parameters, and the differences $\left(X_{2}^{0} \oplus X_{2}^{0}, X_{2}^{1} \oplus X_{2}^{0}, \ldots, X_{2}^{255} \oplus X_{2}^{0}\right)$. Since the ShiftRows, the MixColumns and the AddRoundKey operations are linear, it is sufficient to know the differences $\left(X_{1(S B)}^{0} \oplus X_{1(S B)}^{0}, X_{1(S B)}^{1} \oplus X_{1(S B)}^{0}, \ldots, X_{1(S B)}^{255} \oplus X_{1(S B)}^{0}\right)$.

Now we turn to the "top side" of the four rounds. In round 0 , the differences $\left(X_{0(S B)}^{0} \oplus X_{0(S B)}^{0}, X_{0(S B)}^{1} \oplus X_{0(S B)}^{0}, \ldots, X_{0(S B)}^{255} \oplus X_{0(S B)}^{0}\right)$ are known - these are exactly the 256 possible differences in byte 0 (the rest of the bytes are equal). Note that the order of the differences is not known, but this does not disturb the adversary since in our attack she is interested only in the multiset and not

\footnotetext{
${ }^{4}$ Unlike sets, elements can occur multiple times, and the multiset retains this multiplicity along with the values.
} 
in the sequence. Since the ShiftRows, the MixColumns, and the AddRoundKey operations are linear, the differences $\left(X_{1}^{0} \oplus X_{1}^{0}, X_{1}^{1} \oplus X_{1}^{0}, \ldots, X_{1}^{255} \oplus X_{1}^{0}\right)$ are also known. By the structure of the $\delta$-set, these differences are active in bytes $0,1,2,3$ and passive in the rest of the bytes. Since bytes $0,1,2,3$ of $X_{1}^{0}$ are given as part of the parameters, bytes $0,1,2,3$ of the values $\left\{X_{1}^{1}, \ldots, X_{1}^{255}\right\}$ are thus also known, and so are bytes $0,1,2,3$ of $\left\{X_{1(S B)}^{0}, X_{1(S B)}^{1}, \ldots, X_{1(S B)}^{255}\right\}$. Since the differences $X_{1(S B)}^{j} \oplus X_{1(S B)}^{0}$ in all the bytes except for $0,1,2,3$ are zero for all $j=1,2, \ldots, 255$, this implies that the full vector of differences $\left(X_{1(S B)}^{0} \oplus X_{1(S B)}^{0}, X_{1(S B)}^{1} \oplus X_{1(S B)}^{0}, \ldots, X_{1(S B)}^{255} \oplus X_{1(S B)}^{0}\right)$ is known, as required above.

Finally, since the multiset depends on 24 byte parameters, it can assume at most $2^{192}$ possible values. However, in this count, each $\delta$-set is represented by $2^{8}$ multisets, according to the 256 possible choices of $P^{0}$. We can then reduce the number of parameters by one by choosing $P^{0}$ such that $X_{1,0}^{0}=0$ (this is possible since byte 0 in state $X_{1}$ is active). This reduces the number of possible multisets to $2^{184}$, concluding the proof.

\subsection{The Differential Enumeration Technique}

Observation 4 shows that the possible multisets depend on 24 explicitly stated parameters. In order to reduce the size of the precomputed table, we would like to choose the $\delta$-set such that several of these parameters will equal to predetermined constants. Of course, the key bytes are not known to the adversary and thus cannot be "replaced" by such constants. At first glance, it seems that the bytes in the intermediate states $X_{1}^{0}$ and $X_{2}^{0}$ also cannot be made equal to pre-determined constants by choosing the plaintexts appropriately, since they are separated from the plaintexts by operations involving an unknown key. However, we show that by using an expected-probability differential (i.e., a differential whose probability is not assumed to be especially high or especially low) for 4-round AES, the plaintext $P^{0}$ can be chosen such that the full 128-bit state $X_{2}^{0}$ will assume one of at most $2^{64}$ particular values (which can be computed in advance and are independent of the choice of key) instead of $2^{128}$ possible values.

Consider a truncated differential for four full AES rounds, in which both the input and the output differences are non-zero in a single byte (e.g., byte 0 both in the input and in the output). The probability of this differential is expected to be about $2^{-120} 5$ and thus it is expected that $2^{120}$ randomly chosen pairs with difference only in byte 0 would contain one pair that satisfies the differential. Moreover, since each $\delta$-set contains $2^{15}$ pairs with difference in a single byte, a collection of $2^{105}$ randomly chosen $\delta$-sets in which byte 0 is active is expected to contain a right pair with respect to the differential. For right pairs, we show the following:

\footnotetext{
${ }^{5}$ The probability of $2^{-120}$ is based on the assumption that 4-round AES behaves like a random permutation with respect to this differential, and thus forcing 120 bits to be equal has this probability. If this is not the case, it is expected that other more powerful attacks on AES may exist.
} 


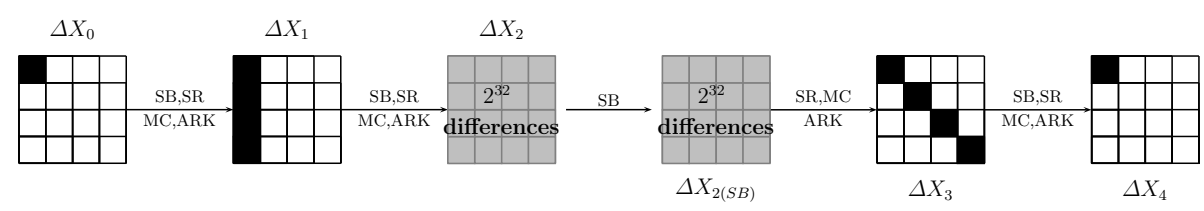

Fig. 3. The 4-Round Differential Characteristic Used in Our Attack

Observation 5. Let $\left(P^{1}, P^{2}\right)$ be a right pair with respect to the differential (i.e., the difference $P^{1} \oplus P^{2}$ is non-zero only in byte 0 , and the difference between the corresponding ciphertexts, $C^{1} \oplus C^{2}$, is also non-zero only in byte 0$)$. Then the intermediate state $X_{2}^{1}$ assumes one of at most $2^{64}$ prescribed values.

\section{Proof}

The proof is a meet-in-the-middle argument. We start with the "top side" of the four rounds. Due to the structure of AES, the difference between the states $X_{1(S B)}^{1}$ and $X_{1(S B)}^{2}$ (i.e., the intermediate values after SubBytes of round 1) is non-zero only in bytes $0,1,2,3$. Thus, this difference can assume at most $2^{32}$ distinct values. Since the ShiftRows, the MixColumns, and the AddRoundKey operations are linear, this implies that the difference $X_{2}^{1} \oplus X_{2}^{2}$ can assume at most $2^{32}$ different values.

On the other hand, from the "bottom side" we see that the difference $X_{3}^{1} \oplus$ $X_{3}^{2}$ is non-zero only in bytes $0,5,10,15$. Since the ShiftRows, the MixColumns, and the AddRoundKey operations are linear, this implies that the difference $X_{2(S B)}^{1} \oplus X_{2(S B)}^{2}$ can assume at most $2^{32}$ different values.

It is well-known that given the input and output differences of the SubBytes operation, there is one possibility on average for the actual pair of input/output values 6 Moreover, this pair of actual values does not depend on the key, and can be easily found by precomputing the full difference distribution table of the SubBytes operation. Since for the right pair we consider, there are at most $2^{32}$. $2^{32}=2^{64}$ possible pairs of input/output differences of the SubBytes operation in round 2 , there are at most $2^{64}$ possible values of the full state $X_{2}^{1}$, as asserted. $\square$

It follows from the observation that if we choose the $\delta$-set such that $P^{0}$ is a member of a right pair with respect to this expected-probability differential, we are assured that the state $X_{2}^{0}$ can assume at most $2^{64}$ possible values. Moreover, since these values do not depend on the key and can be computed in advance, this allows to construct the "table of possible multisets" only for these $2^{64}$ values, which reduces the size of the table and the time complexity of the preprocessing phase by a huge factor of $2^{57}$ as shown below.

Three additional remarks are due:

- Firstly, in order to exploit the expected-probability differential we have to consider as many as $2^{113}$ chosen plain texts, which increases the data

\footnotetext{
${ }^{6}$ Actually, given the input/output differences, with probability of about $1 / 2$ there are no such pairs, with probability of about $1 / 2$ there are two pairs, and with probability of about $1 / 256$ there are four pairs.
} 
complexity of the attack. However, the resultant tradeoff is advantageous since the data complexity was smaller than the other complexities.

- Secondly, in order to detect the right pair with respect to the differential, the adversary has to guess several key bytes in the rounds before and after the differential. However, it turns out that if the differential is chosen such that the non-zero differences are in the bytes which are active in the $\delta$-set, these key bytes coincide with the key bytes that should be guessed in the original Demirci-Selçuk attack. Hence, this does not increase the time complexity of the online phase of the attack.

- Finally, the total number of possible multisets after the combination with the differential is not $2^{184} \cdot 2^{-64}=2^{120}$, but rather $2^{127}$. The reason for this increase is that in the original attack, the number of multisets is reduced by a factor of $2^{8}$ since each $\delta$-set corresponds to $2^{8}$ different multisets, according to the possible choices of $P^{0}$ (see proof of Observation 4). In the new version of the attack, we are forced to choose $P^{0}$ to be one of the members of the right pair w.r.t. the differential, and thus each $\delta$-set corresponds to only two "special" multisets 7 Therefore, the memory complexity and the time complexity of the preprocessing phase are reduced by a factor of $2^{57}$ rather than $2^{64}$, compared to Observation 4 .

\subsection{The Key Bridging Technique}

In this section we show that the time complexity of the online phase in the attacks on 8-round AES-192 and AES-256 can be reduced significantly by using key schedule considerations. While most of these considerations are simple, one of them is a novel observation that allows the adversary to deduce some subkey bytes from some other subkey bytes, even though they are separated by many key mixing steps.

We start with the attack on 8-round AES-192. Recall that in the online phase of this attack, the adversary has to guess four bytes of the subkey $k_{-1}$, one byte of the equivalent subkey $u_{5}$, four bytes of the equivalent subkey $u_{6}$, and the full $k_{7}$. The exact number of bytes that should be guessed depends on the choice of the active byte of the $\delta$-set and of the byte in which the multiset is constructed. It turns out that if the byte to be examined at the end of round 4 is one of the bytes $1,6,11,12$, then the number of guessed key bytes is reduced by three. Indeed, by the key schedule of AES-192, the knowledge of $k_{7}$ yields the

\footnotetext{
${ }^{7}$ We note that while the table of possible multisets is constructed according to one member of the right pair, it may occur that in the actual attack, the other member is chosen as $P^{0}$, and thus the multiset does not match the table (even for the right key guess). A simple solution is to repeat the attack for both members of the right pair. A more advanced solution, which allows to save the extra factor two in the time complexity of the attack, is to store the multisets only up to XOR with a constant value. This can be achieved by a small modification to the preprocessing phase, consisting of XORing each multiset with the 256 possible byte values and storing in the table the resulting multiset which is the least in the lexicographic order amongst the 256 possibilities.
} 
knowledge of the first two columns of $k_{6}$ (and thus also of $u_{6}$ ) and of the last column of $k_{5}$ (and thus also of $u_{5}$ ).

If the byte to be checked at the end of round 4 is byte 1 , then the bytes to guess are byte 13 of $u_{5}$, bytes $3,6,9,12$ of $u_{6}$, and the full subkey $k_{7}$. However, once $k_{7}$ is guessed, bytes 3,6 of $u_{6}$ and byte 13 of $u_{5}$ can be computed from the key schedule, thus reducing the time complexity of the online phase of the attack by a factor of $2^{24}$.

The complexity can be further reduced by another factor of $2^{8}$ using the following novel observation:

Observation 6. By the key schedule of AES-192, knowledge of columns 0,1,3 of the subkey $k_{7}$ allows to deduce column 3 of the whitening key $k_{-1}$ (which is actually Column 3 of the master key).

The main novelty in this observation is that it exploits the weak key schedule of AES-192 in order to provide a surprisingly long "bridge" between two subkeys which are separated by 8 key mixing steps (applied in the reverse direction). In particular, it makes it possible to compute one byte in the whitening subkey $k_{-1}$ directly from four bytes in the last subkey $k_{7} 8$ which saves a factor of $2^{8}$ in the time complexity of any attack which has to guess these five subkey bytes. Since guessing key material in the first and last round is a very common in attack, this observation can be widely applicable (e.g., it can reduce the time complexity of the related-key attack on 8-round AES-192 presented in [14] from $2^{180}$ to $2^{172}$ ).

Proof. For the detailed proof and reasoning, we refer the reader to the full version of the paper. Given $W[32], W[33], W[35]$, it is possible to compute $W[27]=$ $W[32] \oplus W[33]$ and $W[23]=W[33] \oplus W[35]$. From these two values, it is possible to compute $W[3]=W[27] \oplus S B(W[23] \lll 8) \oplus R C O N[4]$.

Since in the 8-round attack, one of the subkey bytes guessed by the adversary is included in the column $W[3]$ (regardless of the active byte in the $\delta$-set), this reduces the time complexity by another factor of $2^{8}$. In total, the key schedule considerations reduce the time complexity of the online phase of the attack on AES-192 by a factor of $2^{32}$.

In the attack on 8-round AES-256, key schedule considerations can help the adversary only a little. By the key schedule, the subkey $u_{6}$ is independent of the subkey $k_{7}$, and thus the only subkey byte the adversary can retrieve is the single byte of $u_{5}$. As the novel observation does not hold for AES-256, key schedule arguments can reduce the time complexity only by a factor of $2^{8}$.

\section{Our New Attack on 7-Round AES}

In this section we present our new attack on 7-round AES. For the sake of simplicity, we present here only the basic variant of the attack, which is used later

${ }^{8}$ The four bytes of $k_{7}$ are 0 and 4 (for obtaining byte 0 of $W[27]$ ) and bytes 7 and 15 (for obtaining byte 3 of $W[23]$ ). 
as part of the 8-round attack. In Appendix $\mathrm{A}$ we show how to improve the attack using alteration of the expected-probability differential and time/memory/data tradeoffs, such that the resulting time complexity will be lower than the complexity of all previously known attacks on 7-round AES (in all its three flavors).

\subsection{The Basic Attack}

In this attack, the byte with non-zero difference in the expected-probability differential is byte 0 , both in the input and in the output differences. The active byte of the $\delta$-set and the byte that is checked in the state $X_{5}$ are taken to be byte 0 as well. The attack works similarly if these bytes are replaced by any other pair of bytes, as long as the correspondence between the differential and the $\delta$-set is preserved.

The algorithm of the basic attack is as follows:

1. Preprocessing phase: Compute the $2^{127}$ possible values of the "special" multisets defined by Observations 4 and 5, and store them in a hash table.

\section{Online phase:}

(a) Phase A - Detecting the right pair

i. Ask for the encryption of $2^{81}$ structures of $2^{32}$ plaintexts, such that in each structure, bytes $0,5,10,15$ assume the $2^{32}$ possible values and the rest of the bytes are constant.

ii. For each structure, store the ciphertexts in a hash table and look for pairs in with no difference in bytes $1,2,3,4,5,6,8,9,11,12,14,159$ Since this is a 96 -bit filtering, about $2^{48}$ pairs remain.

iii. For each remaining pair, guess bytes $0,5,10,15$ of $k_{-1}$ and check whether the difference in the state $X_{1}$ is non-zero only in byte 0 . For each key guess, about $2^{24}$ pairs are expected to remain.

iv. For each remaining pair, guess bytes $0,7,10,13$ of $u_{6}$ and check whether the difference in the state $X_{5}$ is non-zero only in byte 0 . For each key guess, only one pair is expected to remain.

(b) Phase B - Checking the $\delta$-set

i. For each guess of the eight subkey bytes made in Phase A and for the corresponding pair, take one of the members of the pair, denote it by $P^{0}$, and find its $\delta$-set using the knowledge of bytes $0,5,10,15$ of $k_{-1}$. (This is done by taking $X_{1}^{0}$, XORing it with the 255 possible values which are non-zero only in byte 0 , and decrypting the 255 obtained values through round 0 using the known subkey bytes. The resulting plaintexts are the other members of the $\delta$-set.)

ii. Guess byte 0 of $u_{5}$, and using the knowledge of bytes $0,7,10,13$ of $u_{6}$, partially decrypt the ciphertexts of the $\delta$-set to obtain the multiset $\left[X_{5,0}^{0} \oplus X_{5,0}^{0}, X_{5,0}^{1} \oplus X_{5,0}^{0}, \ldots, X_{5,0}^{255} \oplus X_{5,0}^{0}\right]$.

\footnotetext{
${ }^{9}$ In the description of our attack we assume that the last round does not contain the MixColumns operation. If it does contain it, one can swap the order of the last round's MixColumns and AddRoundKey and apply the attack with the respective changes.
} 
iii. Check whether the multiset exists in the hash table. If not, discard the key guess (possibly using auxiliary techniques such as repetition of the attack with a different output byte).

(c) Exhaustively search the rest of the key: For each remaining key guess, find the remaining key bytes by exhaustive search.

It is clear that the time complexity of the online phase of the attack is dominated by encrypting $2^{113}$ plaintexts, and hence, the data and time complexity of this part of the attack is $2^{113}$. The memory complexity is $2^{129} 128$-bit blocks, since each multiset contains about 512 bits of information and its representation can be easily compressed into 512 bits of space. The time complexity of the preprocessing phase of the attack is approximately $2^{127} \cdot 2^{8} \cdot 2^{-3}=2^{132}$ encryptions.

In Appendix A we show that the attack can be improved by altering the expected-probability differential, using several differentials in parallel, and applying time/memory/data tradeoffs. The resulting complexities lie on the following tradeoff curve: Data complexity $-2^{103+n}$ chosen plaintexts, Time complexity $-2^{103+n}$ encryptions, Memory requirement $-2^{129-n}$ AES blocks, for any $n \geq 0$. Choosing $n=13$, all the three complexities are equalized at $2^{116}$, which is lower than the time complexities of all known attacks on 7-round AES, in all its three flavors (see Table 1).

\section{Extension to Attacks on 8-Round AES-192 and AES-256}

In this section we present the first non-marginal attack on 8-round AES-192. The data complexity of the attack is $2^{113}$ chosen plaintexts, the memory requirement is $2^{129} 128$-bit blocks, and the time complexity is $2^{172}$ encryptions. A variant of the attack can be applied to 8-round AES-256. The data and memory requirements remain unchanged, but the time complexity is increased to $2^{196}$ encryptions. We present the attack on AES-192; the attack on AES-256 is similar.

In the attack presented below, we choose the non-zero byte in the output difference of the expected-probability differential to be byte 1 . Accordingly, the byte to be checked in the $\delta$-set is also chosen as byte 1 . This change is required in order to apply the key schedule considerations presented in Section 4.3. The only non-zero byte in the input difference of the differential and the only active byte of the $\delta$-set can be still chosen arbitrarily, as long as they are the same. Without lose of generality, in the sequel we assume that this byte is byte 0 .

A trivial generalization of the 7-round attack presented in Section 5 to eight rounds is to guess the full $k_{7}$, and for each guess, decrypt all the ciphertexts through the last round and apply the 7-round attack. In our attack this approach leads to an extremely high time complexity. Specifically, the detection of the right pair would require $2^{113} \cdot 2^{128}=2^{241}$ encryptions. Instead, we use the early abort technique that was described in [12. We present here the technique only briefly, and refer the reader to 12 for the full details. 
Table 1. A Comparison of Previous Results with Our New Attacks

\begin{tabular}{|c|c|c|c|c|c|c|}
\hline \multicolumn{2}{|c|}{$\overline{\overline{\text { Rounds Key }}}$} & \multicolumn{4}{|c|}{ Complexity } & \multirow{2}{*}{ "Attack Type \& Source } \\
\hline & Size & Data (CP) & Memory & Time & $\operatorname{MinMax}^{\star}$ & \\
\hline \multirow[t]{3}{*}{7} & 128 & $\begin{array}{c}2^{112.2} \\
2^{103+n}\end{array}$ & $\begin{array}{l}2^{112.2} \\
2^{129-n}\end{array}$ & $\begin{array}{c}2^{117.2} \mathrm{MA} \\
\mathbf{2}^{103-n}\end{array}$ & $\begin{array}{c}2^{117.2} \\
2^{116}\end{array}$ & $\begin{array}{l}\text { Impossible Differential [12] } \\
\text { Our Results (Sect. 5) }\end{array}$ \\
\hline & 192 & $\begin{array}{l}19 \cdot 2^{32} \\
2^{32} \\
2^{46+n} \\
2^{113.8} \\
2^{103+n}\end{array}$ & $\begin{array}{c}19 \cdot 2^{32} \\
2^{80} \\
2^{192-n} \\
2^{113.8} \\
\mathbf{2}^{129-n}\end{array}$ & $\begin{array}{c}2^{155} \\
2^{140} \\
2^{94+n} \\
2^{118.8} \mathrm{MA} \\
\mathbf{2}^{103+n}\end{array}$ & $\begin{array}{c}2^{155} \\
2^{140} \\
2^{143} \\
2^{118.8} \\
2^{116}\end{array}$ & $\begin{array}{l}\text { SQUARE [10] } \\
\text { Collision [11] } \\
\text { Meet in the Middle [7] } \\
\text { Impossible Differential [12] } \\
\text { Our Results (Sect 5) }\end{array}$ \\
\hline & 256 & $\begin{array}{c}21 \cdot 2^{32} \\
2^{32} \\
2^{34+n} \\
2^{113.8} \\
2^{103+n} \\
\end{array}$ & $\begin{array}{c}21 \cdot 2^{32} \\
2^{80} \\
2^{204-n} \\
2^{113.8} \\
\mathbf{2}^{129-n} \\
\end{array}$ & $\begin{array}{c}2^{172} \\
2^{140} \\
2^{82+n} \\
2^{118.8} \mathrm{MA} \\
\mathbf{2}^{103+n} \\
\end{array}$ & $\begin{array}{c}2^{172} \\
2^{140} \\
2^{143} \\
2^{118.8} \\
\mathbf{2}^{116} \\
\end{array}$ & $\begin{array}{l}\text { SQUARE [10] } \\
\text { Collision [11] } \\
\text { Meet in the Middle [7] } \\
\text { Impossible Differential [12] } \\
\text { Our Results (Sect 5) }\end{array}$ \\
\hline \multirow[t]{2}{*}{8} & 192 & $\begin{array}{l}2^{127.997} \\
2^{113+n}\end{array}$ & $\begin{array}{c}2^{128} \\
2^{129-n}\end{array}$ & $\begin{array}{c}2^{188} \\
2^{172+n}\end{array}$ & $\begin{array}{l}2^{188} \\
2^{172}\end{array}$ & $\begin{array}{l}\text { SQUARE [10] } \\
\text { Our Results (Sect. 6) }\end{array}$ \\
\hline & 256 & $\begin{array}{c}2^{34+n} \\
2^{34+\max (n-24,0)} \\
2^{89.1} \\
2^{127.997} \\
\mathbf{2}^{113+n}\end{array}$ & $\begin{array}{c}2^{206-n} \\
2^{208-n} \\
2^{97} \\
2^{128} \\
2^{129-n}\end{array}$ & $\begin{array}{c}2^{205.6+n} \\
2^{206+n} \mathrm{MA} \\
2^{229.7} \mathrm{MA} \\
2^{204} \\
\mathbf{2}^{196+n}\end{array}$ & $\begin{array}{c}2^{205.8} \\
2^{208} \\
2^{229.7} \\
2^{204} \\
2^{196}\end{array}$ & $\begin{array}{l}\text { Meet in the Middle }[7]^{\dagger} \\
\text { Meet in the Middle }[8]^{\ddagger} \\
\text { Impossible Differential [12] } \\
\text { SQUARE [10] } \\
\text { Our Results (Sect. 6) }\end{array}$ \\
\hline
\end{tabular}

^ — the lowest time complexity which exceeds the other complexities via the tradeoff option (if available).

${ }^{\dagger}$ - [7] estimates the cost of partial encryption as $2^{-8}$ of an encryption. As there are at least six columns which take part in the partial encryption/decryption, we believe that $2^{-2.4}$ is a more accurate estimate.

$\ddagger$ - The complexity is higher than claimed in [8] due to a flaw in the analysis.

Time complexity measures the time in encryption units unless mentioned otherwise.

Memory complexity is measured in AES blocks.

$\mathrm{CP}$ - Chosen plaintext, MA - Memory Accesses.

In the following, the adversary examines each of the $2^{113} \cdot 2^{31}=2^{144}$ pairs separately, and her goal is to detect the subkey candidates for which that pair satisfies the expected-probability differential.

Note that if $\left(P^{1}, P^{2}\right)$ is a right pair, then the corresponding intermediate states $\left(X_{6(S R)}^{1}, X_{6(S R)}^{2}\right)$ have non-zero difference only in bytes $3,6,9,12$. Hence, in each column of $X_{6(S R)}$ there are only $2^{8}$ possible differences. Since the MixColumns and AddRoundKey operations are linear, this implies that in each column of $X_{7}$ there are only $2^{8}$ possible differences, and thus only $2^{32} \cdot 2^{8}=2^{40}$ possible pairs of actual values. In the technique presented in [12, the adversary considers these $2^{40}$ pairs in advance, encrypts them through round 7 , and stores the actual values before the last AddRoundKey operation in a hash table, sorted by the output difference. In the online phase of the attack, for each examined pair, the adversary considers each shifted column (e.g., bytes $0,7,10,13$ ) independently, and accesses the hash table in the row corresponding to the ciphertext difference. It is expected that $2^{40} \cdot 2^{-32}=2^{8}$ values appear in each row. Since the table gives the actual values before the AddRoundKey operation, and the ciphertexts 
are the values after that operation, each of the pairs in the table suggests one value for the 32-bit subkey corresponding to that shifted column.

Therefore, for each examined pair, and for each shifted column, the adversary obtains a list of $2^{8}$ candidates for the 32 -bit subkey corresponding to that column. In a basic variant of the attack, the adversary aggregates these suggestions to $2^{32}$ suggestions for the full $k_{7}$, and for each suggestion, she decrypts the ciphertext pair through round 7 . Then she uses a similar precomputed table for round 6 to get a list of $2^{8}$ possible values of bytes $3,6,9,12$ of $u_{6}$. For each such value, the adversary checks whether the relations between bytes 3,6 of $u_{6}$ and the subkey $k_{7}$ described in Section 4.3 hold. If not, the subkey guess is discarded. Since this is a 16-bit filtering, the adversary is left with $2^{24}$ candidates for the full $k_{7}$ and bytes $3,6,9,12$ of $u_{6}$. Finally, using a precomputed table also in round 0 , the adversary obtains a list of $2^{8}$ possible values of bytes $0,5,10,15$ of $k_{-1}$. For each such value, the adversary checks whether the relation between byte 15 of $k_{-1}$ and the subkey $k_{7}$ described in Section 4.3 holds. If not, the subkey guess is discarded. Since this is an 8-bit filtering, the adversary is left with $2^{24}$ candidates for the full $k_{7}$, bytes $3,6,9,12$ of $u_{6}$, and bytes $0,5,10,15$ of $k_{-1}$. For each of these candidates, $\left(P^{1}, P^{2}\right)$ is a right pair w.r.t. the expected-probability differential, and the second-phase of the attack can be applied.

The time complexity of this procedure is $2^{40}$ simple operations for each examined pair, or $2^{144} \cdot 2^{40} \cdot 2^{-8}=2^{176}$ encryptions in total.

The time complexity can be slightly reduced by using a more sophisticated precomputed table in order to check the consistency between bytes 3,6 of $u_{6}$ and the subkey $k_{7}$. The table takes bytes 3,6 of $M C^{-1}\left(X_{6}\right)$ in both pairs, along with bytes $2,3,5,6$ of $u_{7}$, and returns the consistent values for bytes 3,6 of $u_{6}$, if there are any. The precomputation is done by trying all possible candidates for the pair of bytes for $M C^{-1}\left(X_{6}\right)$ along with the corresponding bytes of $u_{6}$, to see if the decrypted values satisfy the linear relation on the differences before the SubBytes operation of round 5. If this is the case, the entry corresponding to the $M C^{-1}\left(X_{6}\right)$ values and all subkeys of $u_{7}$ which satisfy the key relation is stored with the respective $u_{6}$ bytes. We note that for each key and each pair, there is probability of $2^{-8}$ that the condition is satisfied, and thus, only $2^{56}$ of the entries in the table are nonempty.

At the second part of the online phase of the attack, performed for each of the $2^{144}$ pairs $\left(P^{1}, P^{2}\right)$ and each of the $2^{24}$ subkeys corresponding to the pair, the adversary constructs a $\delta$-set and checks whether the corresponding multiset appears in the table. Note that while in the 7-round attack this phase requires guessing an additional subkey byte (which is byte 13 of $u_{5}$ ), in this attack that subkey byte can be derived from the subkey $k_{7}$. The time complexity of the second part is $2^{168} \cdot 2^{8} \cdot 2^{-4}=2^{172}$ encryptions.

Therefore, the overall memory requirement of the attack is $2^{129} 128$-bit blocks (as in the basic version of the 7 -round attack), the data complexity is $2^{113}$ chosen plaintexts, and the time complexity is $2^{172}$ encryptions. These complexities improve significantly over the only previously known attack on AES-192, which is a 
SQUARE attack [10] requiring almost the entire codebook and time complexity of $2^{188}$ encryptions.

\section{Summary}

In this paper we introduced three new cryptanalytic techniques which can be used to improve the best known complexities of all the known attacks on 7 and 8 round versions of AES, as detailed in Table 1, In particular, we describe the first real attack on 8-round AES-192 which does not use the full codebook in order to marginally improve the time complexity of exhaustive search. However, all our attacks have impractical complexities, and thus they do not endanger the security of any fielded system.

\section{References}

1. Bahrak, B., Aref, M.R.: Impossible Differential Attack on 7-round AES-128. IET (IEE). J. on Information Security 2(2), 28-32 (2008)

2. Biryukov, A., Dunkelman, O., Keller, N., Khovratovich, D., Shamir, A.: Key Recovery Attacks of Practical Complexity on AES-256 Variants With Up To 10 Rounds. In: Gilbert, H. (ed.) Advances in Cryptology - EUROCRYPT 2010. LNCS, vol. 6110, pp. 299-319. Springer, Heidelberg (2010)

3. Biryukov, A., Khovratovich, D.: Related-Key Cryptanalysis of the Full AES-192 and AES-256. In: Matsui, M. (ed.) ASIACRYPT 2009. LNCS, vol. 5912, pp. 1-18. Springer, Heidelberg (2009)

4. Biryukov, A., Khovratovich, D., Nikolic, I.: Distinguisher and Related-Key Attack on the Full AES-256. In: Halevi, S. (ed.) Advances in Cryptology - CRYPTO 2009. LNCS, vol. 5677, pp. 231-249. Springer, Heidelberg (2009)

5. Daemen, J., Rijmen, V.: AES Proposal: Rijndael, AES proposal (1998)

6. Daemen, J., Rijmen, V.: The design of Rijndael: AES - the Advanced Encryption Standard. Springer, Heidelberg (2002)

7. Demirci, H., Selçuk, A.A.: A Meet-in-the-Middle Attack on 8-Round AES. In: Nyberg, K. (ed.) FSE 2008. LNCS, vol. 5086, pp. 116-126. Springer, Heidelberg (2008)

8. Demirci, H., Taskin, I., Çoban, M., Baysal, A.: Improved Meet-in-the-Middle Attacks on AES. In: Roy, B., Sendrier, N. (eds.) INDOCRYPT 2009. LNCS, vol. 5922, pp. 144-156. Springer, Heidelberg (2009)

9. Dunkelman, O., Keller, N.: A New Attack on the LEX Stream Cipher. In: Pieprzyk, J. (ed.) ASIACRYPT 2008. LNCS, vol. 5350, pp. 539-556. Springer, Heidelberg (2008)

10. Ferguson, N., Kelsey, J., Lucks, S., Schneier, B., Stay, M., Wagner, D., Whiting, D.: Improved Cryptanalysis of Rijndael. In: Schneier, B. (ed.) FSE 2000. LNCS, vol. 1978, pp. 213-230. Springer, Heidelberg (2001)

11. Gilbert, H., Minier, M.: A collision attack on 7 rounds of Rijndael. In: Proceedings of the Third AES Candidate Conference (AES3), New York, USA, pp. 230-241 (2000)

12. Lu, J., Dunkelman, O., Keller, N., Kim, J.: New Impossible Differential Attacks on AES. In: Chowdhury, D.R., Rijmen, V., Das, A. (eds.) INDOCRYPT 2008. LNCS, vol. 5365, pp. 279-293. Springer, Heidelberg (2008) 
13. US National Institute of Standards and Technology, Advanced Encryption Standard, Federal Information Processing Standards Publications No. 197 (2001)

14. Zhang, W., Wu, W., Zhang, L., Feng, D.: Improved Related-Key Impossible Differential Attacks on Reduced-Round AES-192. In: Biham, E., Youssef, A.M. (eds.) SAC 2006. LNCS, vol. 4356, pp. 15-27. Springer, Heidelberg (2007)

\section{A Improvements of the Attack on 7-Round AES}

\section{A.1 Altering the Expected-Probability Differential}

Our first improvement reduces the data and time complexities of the attack by a factor of $2^{8}$ without affecting the memory requirements.

We observe that the time complexity of most components of the attack is significantly lower than the time required to encrypt the plaintexts. Therefore, a tradeoff that would decrease the data complexity, even at the price of increasing the time complexity of the other parts of the attack, may reduce its overall complexity.

Such tradeoff is achieved by slightly modifying the expected-probability differential used in the attack. Instead of requiring the input difference to be non-zero only in byte 0 , we can allow the difference to be non-zero also in one of the bytes $5,10,15$. These bytes are chosen such that the number of possible differences in the state $X_{2}$ is not increased, and thus the memory complexity is preserved.

This change reduces the data complexity of the attack to $2^{105}$, since it allows the adversary to use structures of size $2^{16}$ that contain $2^{31}$ pairs with the input difference of the differential. On the other hand, the change requires to guess four additional bytes of $k_{-1}$ in order to detect the right pair (if the additional byte is byte 5 , then the additional guessed bytes are $3,4,9,14)$. As a result, the number of pairs remaining after the first filtering step of the attack is increased to $2^{72}$ (instead of $2^{48}$ ). For each such pair, there are $2^{24}$ possible values of 12 subkey bytes ( 8 bytes of $k_{-1}$ and 4 bytes of $u_{6}$ ) for which that pair satisfies the expected-probability differential. As in the 8-round attack, these values can be found with time complexity of $2^{24}$ table look-ups for each pair, using the early abort technique. Thus, the time complexity of Phase A of the modified attack is $2^{96}$ table look-ups.

In Phase B, we observe that since the value of bytes $3,4,9,14$ of $k_{-1}$ is irrelevant to the examination of the $\delta$-set, the phase has to be performed only $2^{16}$ times for each of the $2^{72}$ pairs (instead of $2^{24}$ times). Thus, its time complexity is $2^{72} \cdot 2^{16} \cdot 2^{8} \cdot 2^{8} \cdot 2^{-3}=2^{101}$ encryptions. Therefore, the overall time complexity of the attack is still dominated by the encryption of the plaintexts, and thus both the data and the time complexity of the attack are reduced to $2^{105}$.

\section{A.2 Using Several Differentials in Parallel}

Our second improvement further reduces the data and time complexities by a factor of 5 without affecting the memory requirements. 
We observe that the data complexity can be reduced by using several differentials in parallel. Since there is no specialty in the choice of the active byte at the input and the output of the original differential, there are 256 possible differentials that can be used in parallel. In the basic 7-round attack this improvement leads to a data/memory tradeoff: The attack requires the "active" bytes of the $\delta$-set to correspond to the non-zero difference bytes of the differential, and altering the active bytes of the $\delta$-set requires preparing a different precomputed table for each choice of the bytes. As a result, the data complexity can be reduced by factor of up to 256, but the memory requirement is increased by the same factor. Since the memory complexity is the dominant one in the 7-round attack, this tradeoff is not profitable.

However, in the modified attack the data complexity can be reduced by a small factor without affecting the memory complexity. We observe that since the additional "active" byte in the expected-probability differential is not used in the analysis of the $\delta$-set, it can be chosen without affecting the memory complexity. There are six possible ways to choose this byte (bytes 5, 10, 15 in the input and bytes 1,2,3 in the output), and five of them can be used in parallel with the same set of chosen plaintexts 10 This reduces the data complexity of the attack by a factor of 5 without affecting the memory complexity. Since the time complexity is dominated by encrypting the plaintexts, it is also reduced by a factor of 5. Therefore, the data and time complexities of the modified attack are smaller than $2^{103}$. In the sequel, we assume for the sake of simplicity that these complexities are equal to $2^{103}$.

\section{A.3 Time/Memory/Data Tradeoffs}

We conclude with a fine tuning of the complexities using a simple tradeoff between data, time, and memory as proposed in [7]. In the preprocessing phase, we precompute the table only for some of the values, and then for each key guess, we perform the attack for several $\delta$-sets in order to compensate for the missing part of the table. For each $n \geq 0$, this tradeoff decreases the memory complexity and the time complexity of the preprocessing phase by a factor of $2^{n}$, and increases the data complexity and the online time complexity by the same factor $2^{n}$. The resulting complexities lie on the following tradeoff curve: Data complexity $-2^{103+n}$ chosen plaintexts, Time complexity $-2^{103+n}$ encryptions, Memory requirement $-2^{129-n}$ AES blocks, for any $n \geq 0$. Choosing $n=13$, all the three complexities are equalized at $2^{116}$, which is lower than the time complexities of all known attacks on 7-round AES, in all its three flavors.

${ }^{10}$ In order to do this, the adversary considers structures of size $2^{96}$ plaintexts each, in which bytes $1,6,11,12$ are constant and the other bytes take all the $2^{96}$ possible values. This allows to use bytes 5 and 10 as the additional active byte in the input of the differential. 